\title{
Population pharmacokinetics and dosing optimization of cefathiamidine in children with hematologic infection
}

This article was published in the following Dove Press journal:

Drug Design, Development and Therapy

\author{
Li-Juan Zhi ${ }^{1,2, *}$ \\ Li Wang ${ }^{2,3, *}$ \\ Xing-Kai Chen ${ }^{4}$ \\ Xiao-Ying Zhai ${ }^{3}$ \\ Li Wen ${ }^{3}$ \\ Lei Dong ${ }^{1,2}$ \\ Evelyne Jacqz-Aigrain ${ }^{5,6}$ \\ Zhong-Ren $\mathrm{Shi}^{2, *}$ \\ Wei Zhao ${ }^{1,2,4, *}$
}

'Department of Pharmacy, Children's Hospital of Hebei Province, Shijiazhuang, People's Republic of China; ${ }^{2}$ Pediatric Research Institute, Children's Hospital of Hebei Province, Shijiazhuang, People's Republic of China; ${ }^{3}$ Department of Pediatric Hematology-Oncology, Children's Hospital of Hebei Province, Shijiazhuang, People's Republic of China; ${ }^{4}$ Department of Clinical Pharmacy, School of Pharmaceutical Sciences, Shandong University, Jinan, People's Republic of China; ${ }^{5}$ Department of Pediatric Pharmacology and Pharmacogenetics, Hôpital Robert Debré, APHP, Paris, France; ${ }^{6}$ Clinical Investigation Center CICI426, INSERM, Paris, France

*These authors contributed equally to this work

Correspondence: Zhong-Ren Shi; Wei Zhao

Pediatric Research Institute, Children's Hospital of Hebei Province, Shijiazhuang 050000, People's Republic of China Tel +86 3।I 859। I204 Email hbseryyeys@163.com; zhao4wei2@hotmail.com
Purpose: Cefathiamidine, a first-generation cephalosporin, has approval from the China Food and Drug Administration for the treatment of infections caused by susceptible bacteria in both adults and children. As pharmacokinetic data are limited in the pediatric population, we aimed to evaluate the population pharmacokinetics of cefathiamidine in children and to define the appropriate dose in order to optimize cefathiamidine treatment.

Methods: Blood samples were collected from children treated with cefathiamidine, and concentrations were quantified by high-performance liquid chromatography and tandem mass spectrometry. Population pharmacokinetic analysis was conducted using NONMEM software. Results: Fifty-four children (age range: 2.0-11.8 years) were included. Sparse pharmacokinetic samples $(n=120)$ were available for analysis. A two-compartment model with first-order elimination showed the best fit with the data. A covariate analysis identified that bodyweight had a significant impact on cefathiamidine pharmacokinetics. Monte Carlo simulation demonstrated that the currently used dosing regimen of $100 \mathrm{mg} / \mathrm{kg} /$ day q12h was associated with a high risk of underdosing in pediatric patients. To reach the target $70 \% \mathrm{fT}>\mathrm{MIC}$, a dose of $100 \mathrm{mg} / \mathrm{kg} / \mathrm{day}$ cefathiamidine $\mathrm{q} 6 \mathrm{~h}$ is required for effective treatment against Haemophilus influenzae.

Conclusion: A population pharmacokinetics model of cefathiamidine in children with hematologic disease was established. A dosing regimen of $100 \mathrm{mg} / \mathrm{kg} /$ day cefathiamidine $\mathrm{q} 6 \mathrm{~h}$ should be used in clinical practice against $H$. influenza infections.

Keywords: cefathiamidine, pharmacokinetics, dosing, children

\section{Introduction}

Cefathiamidine, a first-generation cephalosporin, is approved by the China Food and Drug Administration for the treatment of adults and children with infections due to susceptible bacteria. ${ }^{1}$ Pharmacoepidemiology studies have demonstrated that cefathiamidine is one of the most commonly used cephalosporins in Chinese pediatric hospitals. ${ }^{2,3}$ According to the database of the China Medical Information Centre, cefathiamidine was the third most frequently prescribed agent in the list of the most commonly used antimicrobials in 2012. It has a wide spectrum of antimicrobial activity against Streptococcus pneumoniae, Streptococcus pyogenes, Haemophilus influenzae, Methicillin-sensitive Staphylococcus aureus (MSSA), Methicillin-sensitive Staphylococcus epidermidis (MSSE), and Branhamella (moraxella) catarhalis. ${ }^{4,5}$

Cefathiamidine is not absorbed orally and is, thus, administered through the parenteral route (intravenously or intramuscularly). It is widely distributed in most bodily fluids and tissues; however, it cannot pass through the blood-brain barrier. 
The protein-binding capacity of cefathiamidine is $23 \%$, and more than $90 \%$ of cefathiamidine is excreted unchanged by the kidney. ${ }^{5,6}$ Although cefathiamidine has been included in the standard care for pediatric antimicrobial therapy, there is a lack of pharmacokinetic data in children, which might result in an improper dosing regimen, contributing thereby to risks of failure of antimicrobial therapy and emergence of antibiotic resistance in this vulnerable population. Thus, the objectives of this study were to evaluate the pharmacokinetics of cefathiamidine in children by using a population approach and to establish a simulation-based dosing regimen of cefathiamidine in this vulnerable population.

\section{Methods Study design}

This trial was a prospective, open-label pharmacokinetic study of cefathiamidine and was conducted at the Children's Hospital, Hebei Province. The inclusion criteria were as follows: children aged between 2 and 12 years with confirmed or suspected bacterial infection and cefathiamidine used as a part of regular treatment of bacterial infection. The exclusion criteria were as follows: expected survival time less than the treatment cycle; patients enrolled in a clinical trial; patients with other factors that the researcher considered unsuitable for inclusion. This study was designed in accordance with legal requirements and the Declaration of Helsinki and was approved by the institutional ethics board (Children's Hospital of Hebei Province, approval no 100). Written informed consent was obtained from parents or guardians of patients.

\section{Dosing regimen and pharmacokinetic sampling}

Cefathiamidine $100 \mathrm{mg} / \mathrm{kg} /$ day (Xianlisu ${ }^{\circledR}$, Guangzhou Baiyunshan Pharmaceuticals, Guangzhou, People's Republic of China) was administered $\mathrm{q} 12 \mathrm{~h}$ as an intravenous infusion. The total number of study-specific blood samples was restricted to two per participant. Patients were randomly assigned to either of the two predefined, sparse, pharmacokinetic sampling schedules: Group 1: the end of infusion (3-5 minutes) and 4-8 hours after the start of infusion; and Group 2: 1-2 and 8-12 hours after the start of infusion. Precise infusion and sample times were recorded. Samples of $0.2 \mathrm{~mL}$ were obtained for pharmacokinetic analyses at each sampling. Blood samples were refrigerated and centrifuged, and the plasma was stored at $-80^{\circ} \mathrm{C}$. Processed samples were shipped on dry ice to the Department of Clinical Pharmacy at Shandong Provincial Qianfoshan Hospital, where they were stored at $-80^{\circ} \mathrm{C}$ prior to analysis.

\section{Analytical methods for cefathiamidine}

Cefathiamidine concentrations were detected on an ultraperformance liquid chromatography tandem mass spectrometry (UPLC MS/MS) system comprising a API 4000 triple quadrupole mass spectrometer equipped with an electrospray ionization (ESI) source (Framingham, MA, USA). A series Waters UPLC system (Waters, USA) - consisting of a binary pump, an automatic sampler, and an online degasser - were used for LC-MS/MS analysis. Separation was achieved by using an ACQUITY UPLC ${ }^{\circledR}$ BEH C18 column $(1.7 \mu \mathrm{m}$, $2.1 \times 50 \mathrm{~mm}$ ) purchased from Waters (USA). The mobile phase consisted of water and methanol at a flow rate of $0.4 \mathrm{~mL} / \mathrm{min}$. Determination of cefathiamidine was based on the internal standard method, using ceftiofur as the internal standard (IS). The multiple reaction monitoring transitions of $\mathrm{m} / \mathrm{z} 473.5^{+} \rightarrow 201.3^{+}$and $524.3^{+} \rightarrow 241.4^{+}$were used to quantify cefathiamidine and IS, respectively. The heated nebulizer was set at $300^{\circ} \mathrm{C}$, and the needle voltage (IS) was set at $4,000.00 \mathrm{~V}$; the nebulizer gas (GS1), auxiliary nitrogen gas (GS2), curtain gas (CUR), and collision gas (CAD) were set at $20 \mathrm{psi}, 20 \mathrm{psi}, 10 \mathrm{psi}$, and medium, respectively. Nitrogen was used as the collision gas. The calibration curve ranged from 30 to $10,000 \mathrm{ng} / \mathrm{mL}$. The interday and intraday coefficients of variation of controls were less than $5 \%$. The lower limit of quantification was $30 \mathrm{ng} / \mathrm{mL}$.

\section{Population pharmacokinetic modeling of cefathiamidine}

The nonlinear mixed effects modeling program (NONMEM v7.2, Icon Development Solutions, Columbia, MD, USA) was used for pharmacokinetic analysis. The estimation of pharmacokinetic parameters and their variabilities was carried out using a first-order conditional estimation method with an interactive option.

An exponential model was selected to estimate the interindividual variability of the pharmacokinetic parameter and could be expressed as follows:

$$
\Theta_{\mathrm{i}}=\theta_{\text {mean }} * \mathrm{e}^{\eta \mathrm{i}}
$$

where $\eta \mathrm{i}$ represents the individual pharmacokinetic parameter value of the ith subject; $\theta_{\text {mean }}$ and $\theta_{\mathrm{i}}$ are the typical values of the parameter in the population and the variability between subjects (assumed to follow a normal distribution), respectively.

Covariate analysis used a forward and backward selection method. Stepwise covariate modeling and likelihood ratio test were used to evaluate the potential impact of each covariate relationship on model parameters. During the 
forward selection step, a covariate was included into the model if a significant $\left(p<0.05, \chi^{2}\right.$ distribution with one degree of freedom) decrease (reduction $>3.84$ ) in the objective function value (OFV) from the base model was achieved. Then, all the significant covariates were added simultaneously into the full model. At the backward selection step, the contribution of each significant covariate was removed one by one from the full model and was again retained in the final model if a significant increase in the OFV was obtained.

Both graphical and statistical criteria were used for model validation. Goodness-of-fit plots, including observed observed concentrations (DV) versus population prediction (PRED), DV versus individual prediction (IPRED), conditional weighted residuals (CWRES) versus time, and CWRES versus PRED, were initially used for diagnostic purposes. $^{7}$ Moreover, the stability and performance of the final model was evaluated by a nonparametric bootstrap with re-sampling and replacement 1,000 times using PsN (v2.30). Furthermore, the final model was evaluated graphically and statistically by normalized prediction distribution errors (NPDEs). ${ }^{8,9}$ In total, 1,000 data sets were simulated using the final population model parameters. The NPDE results are shown graphically by the NPDE R package (v1.2), ${ }^{10}$ including a QQ plot and histogram of the NPDE. The NPDE is assumed to follow normal distribution.

\section{Dosing regimen evaluation and optimization based on a pharmacokinetic model}

The pharmacokinetic-pharmacodynamic relationship of cephalosporin is the duration ( $\mathrm{T}$ ) of free antimicrobial drug concentration above the minimum inhibitory concentration
(fT $>$ MIC). For the maximal bactericidal activity of a cephalosporin, a probability of target attainment of $70 \%$ was required for the plasma concentrations during the times of the dosing interval when the $\mathrm{T}_{\mathrm{MIC}}$ was $70 \%{ }^{11-13}$

The free fraction of cefathiamidine was reported to be $0.77 .{ }^{4}$ S. pyogenes, H. influenza, and MSSA (MICs of 0.5, 2, and $8 \mathrm{mg} / \mathrm{L}$, respectively, for susceptible isolates) can cause severe and fatal infections in children. Furthermore, $S$. pneumoniae is a common pathogen but is very sensitive to cefathiamidine, with an MIC of $0.25 \mathrm{mg} / \mathrm{L} .{ }^{14}$ Monte Carlo simulations were undertaken using the parametric estimates obtained from the final model. The pediatric dose of cefathiamidine was simulated on a milligram per kilogram basis. One hundred simulations were carried out using the original data set, and the time above the MIC was calculated for each simulated patient. If the current dosing regimen showed underdosing in the majority of patients, the optimal dosing regimen with an increased dose and/or frequency was given to the virtual patient. ${ }^{15-17}$ The probability of target attainment was then calculated for each dosing regimen to optimize antimicrobial therapy.

\section{Results Study population}

Fifty-four patients were included from April 2015 to March 2016. All patients fulfilled the inclusion and exclusion criteria. All of them received $100 \mathrm{mg} / \mathrm{kg} /$ day cefathiamidine q12h. The mean (SD; range) age and bodyweight of the 54 patients at the time of study were $5.2(2.4 ; 2.0-11.8)$ years and $18.4(6.5 ; 8.0-36.0) \mathrm{kg}$, respectively. Age and bodyweight were all normally distributed ( $p=0.69$ and $p=0.34$, respectively, Kolmogorov-Smirnov test). A summary of patient characteristics is presented in Table 1.

Table I Baseline characteristics in 54 children

\begin{tabular}{|c|c|c|c|}
\hline Characteristics & Number & Mean (SD) & Median (range) \\
\hline Patients & 54 & & \\
\hline Gender & 37 males/ 17 females & & \\
\hline Samples & 120 & & \\
\hline Age (years) & & $5.2(2.4)$ & $4.9(2.0-11.8)$ \\
\hline Current weight (kg) & & $18.4(6.5)$ & $17.8(8.0-36.0)$ \\
\hline Serum creatinine concentration $(\mu \mathrm{mol} / \mathrm{L})$ & & $27(8)$ & $27(10-47)$ \\
\hline Creatinine clearance $(\mathrm{mL} / \mathrm{min})$ & & $211(70)$ & $193(130-462)$ \\
\hline Cefathiamidine dose (mg/dose) & & $910(310)$ & $950(500-1,800)$ \\
\hline Cefathiamidine dose (mg/kg/dose) & & 50 & $50(28-112)$ \\
\hline \multicolumn{4}{|l|}{ Hematologic disease } \\
\hline Leukemia & 23 & & \\
\hline Immune thrombocytopenia & 10 & & \\
\hline Infectious mononucleosis syndrome & 2 & & \\
\hline Anemia & 9 & & \\
\hline Neuroblastoma & 2 & & \\
\hline Hemophagocytic syndrome & 2 & & \\
\hline Others & 6 & & \\
\hline
\end{tabular}




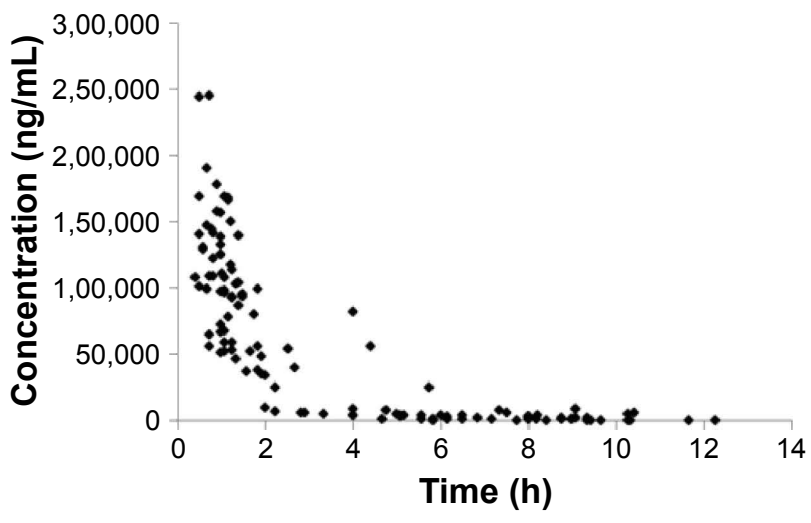

Figure I Cefathiamidine concentrations versus time.

\section{Model building}

In total, 120 cefathiamidine concentrations were available for population pharmacokinetic modeling. The cefathiamidine concentrations of pharmacokinetic samples ranged from 65 to $245,000 \mathrm{ng} / \mathrm{mL}$. The concentration versus time profile is shown in Figure 1.

A two-compartment model with first-order elimination fitted the data well. The model was parameterized in terms of volume of central volume of distribution $\left(\mathrm{V}_{1}\right)$, peripheral volume of distribution $\left(\mathrm{V}_{2}\right)$, intercompartmental clearance $(\mathrm{Q})$, and clearance (CL) of cefathiamidine. The interindividual variability was best described by an exponential model and was then estimated for $\mathrm{CL}$ and $\mathrm{V}_{2}$. This exponential model best described the residual variability.

\section{Covariate analysis}

The allometric size approach was used by incorporating, a priori, the body weight into the base model (allometric coefficients of 0.75 for $\mathrm{CL}$ and $\mathrm{Q}$, and 1 for $\mathrm{V}_{1}$ and $\mathrm{V}_{2}$ ), which caused a significant drop of 16.30 points in the OFV. However, age and creatinine clearance concentration, separately, did not produce a significant decrease in OFV. Therefore, only the effect of bodyweight on $\mathrm{CL} / \mathrm{F}$ and $\mathrm{V}_{2} / \mathrm{F}$ was found to be significant.

The median (range) of estimated weight-normalized CL and volume distribution at steady state (sum of $\mathrm{V}_{1}$ and $\mathrm{V}_{2}$ ) were $0.25(0.05-0.43) \mathrm{L} / \mathrm{h} / \mathrm{kg}$ and $0.31(0.26-0.38) \mathrm{L} / \mathrm{kg}$, respectively. The $\mathrm{AUC}_{0-24}$ at steady state for the evaluated dose regimen ranged from 120 to $550 \mathrm{mg} \cdot \mathrm{h} / \mathrm{L}$. Cefathiamidine CL increased allometrically with weight in children (Figure 2).

\section{Model evaluation}

Model diagnostics showed acceptable goodness-of-fit for the final model of cefathiamidine. As shown in Figure 3A and $\mathrm{B}$, predictions are unbiased. In the diagnostic plots of CWRES versus time and PRED, no trends were observed (Figure 3C and D). Moreover, the mean pharmacokinetic parameters obtained from the bootstrap were in agreement with the respective values estimated from the final model, indicating that the final model was stable and the estimated parameters were accurate (Table 2). NPDE results are presented in Figure 3E and F. The mean and variance of NPDE were -0.0723 (Wilcoxon signed-rank test $p=0.424$ ) and 1.03 (Fisher variance test 0.789 ), respectively, indicating a good fit of the model to the individual data.

\section{Dosing regimen evaluation and optimization}

The target attainment rates as a function of dose for different organisms are shown in Figure 4. With the dosing

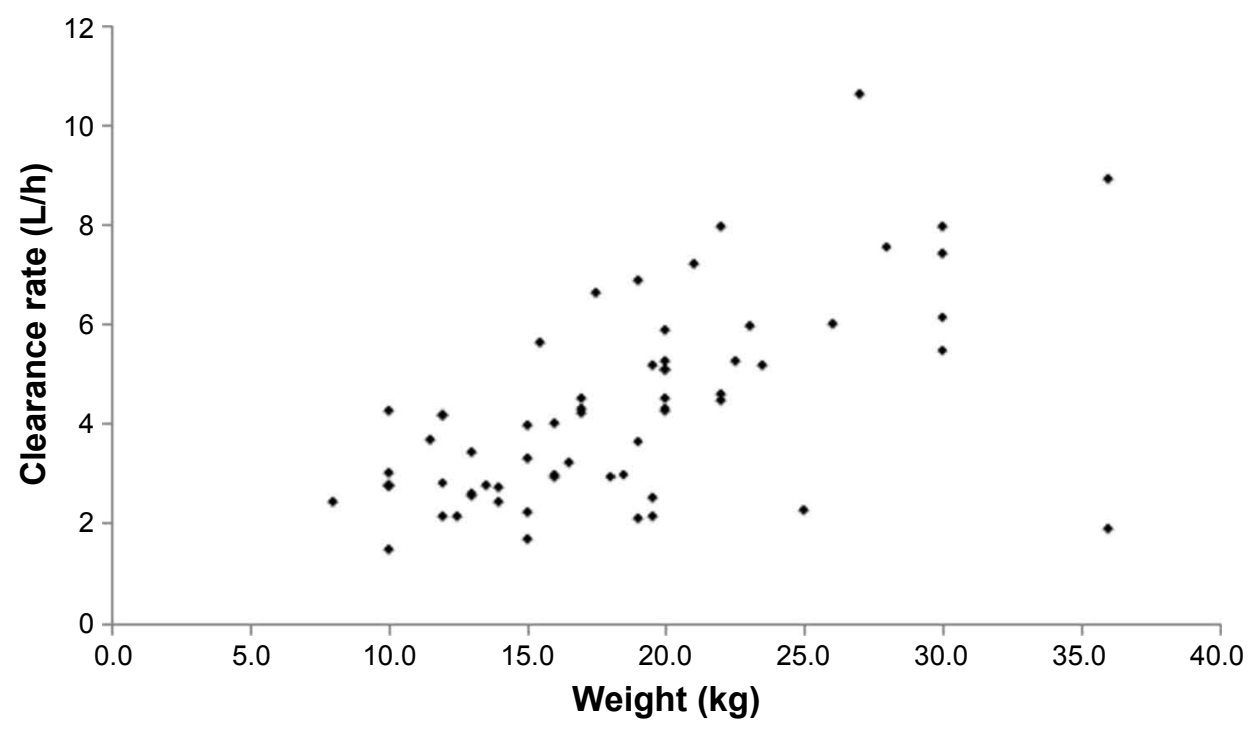

Figure 2 Weight versus cefathiamidine clearance $(C L)$. 

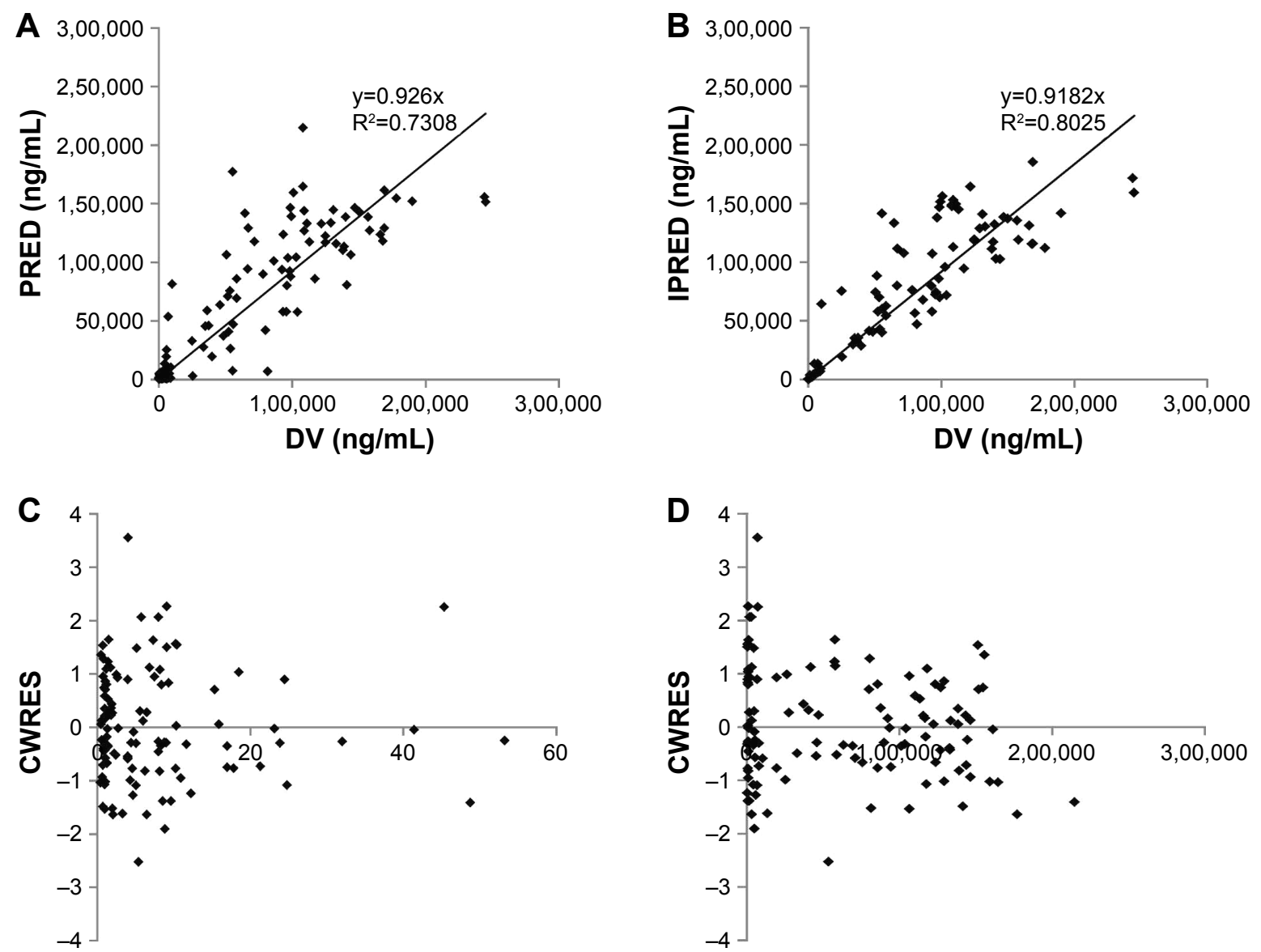

Time since starting treatment (h)
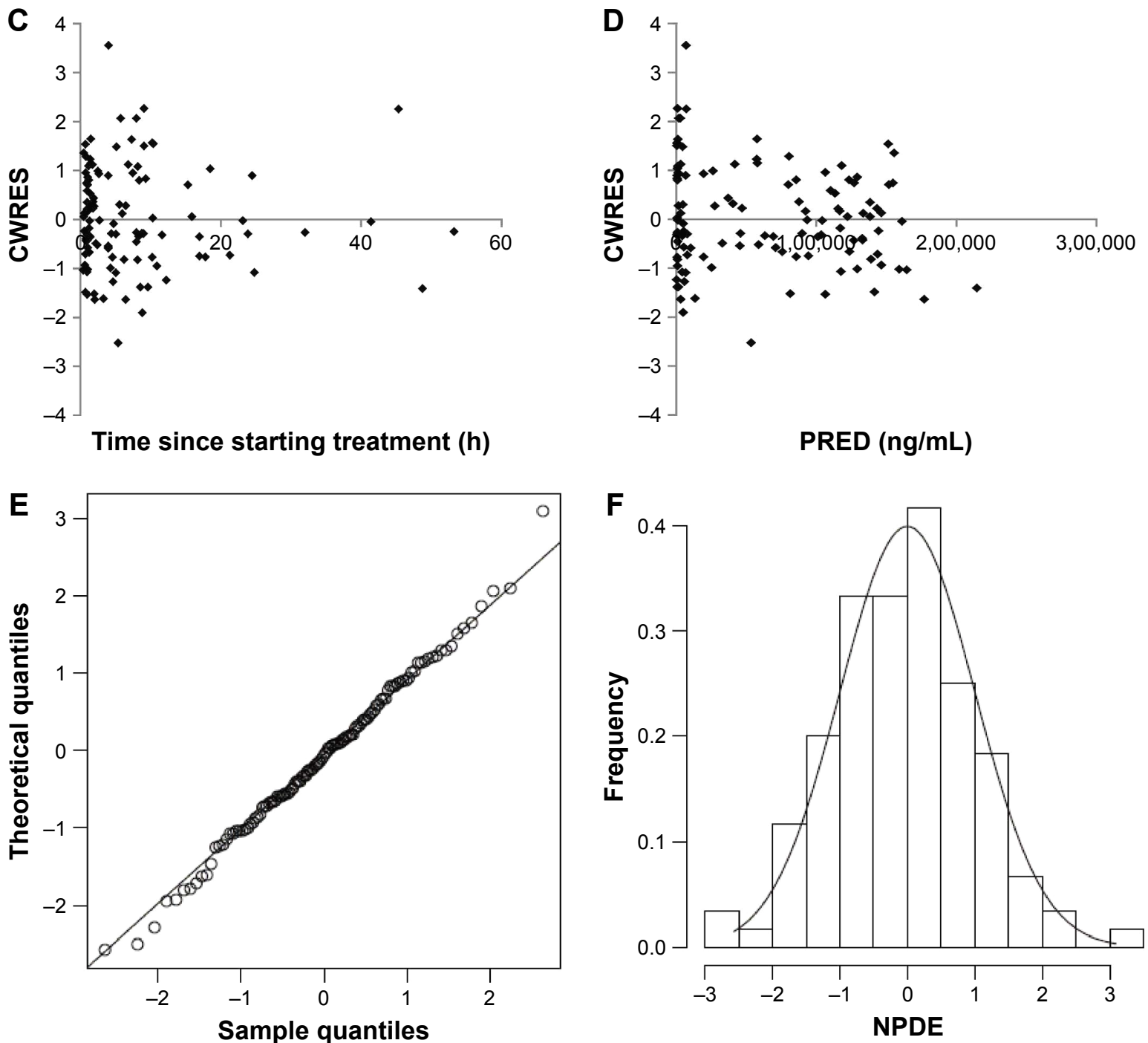

PRED (ng/mL)

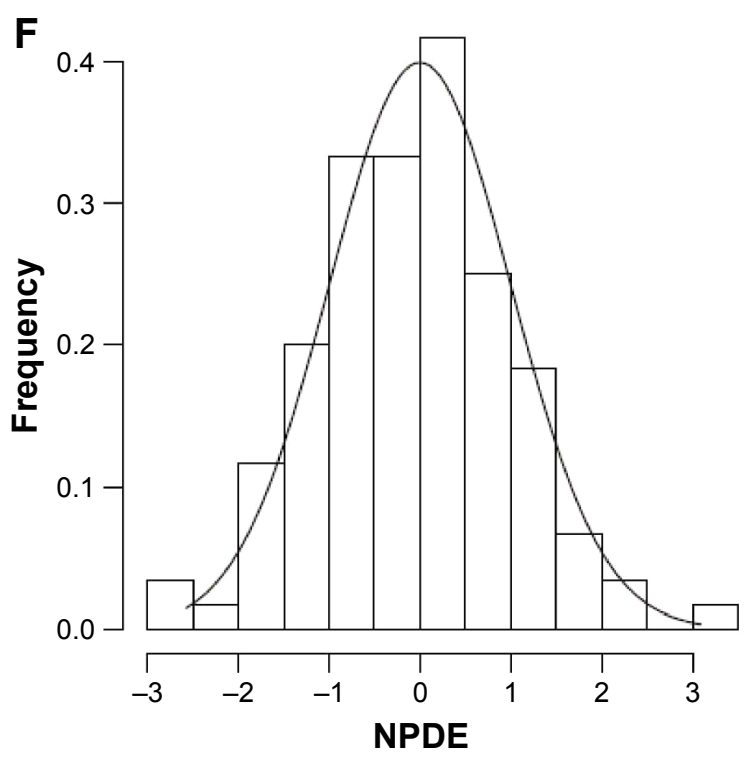

Figure 3 Model evaluation for cefathiamidine. (A) Population predicted (PRED) versus observed concentrations (DV); (B) individual predicted (IPRED) versus DV; (C) conditional weighted residuals (CWRES) versus time; (D) CWRES versus PRED; (E) QQ plot of the distribution of the normalized prediction distribution errors (NPDEs) versus the theoretical N $(0, I)$ distribution; $(\mathbf{F})$ histogram of the distribution of the NPDE, overlaid with the density of the standard Gaussian distribution.

regimen prescribed in the study $(100 \mathrm{mg} / \mathrm{kg} / \mathrm{day} \mathrm{q} 12 \mathrm{~h})$, $67.2 \%$ of children achieved the pharmacodynamic target $(70 \%$ fT $>$ MIC) against the sensitive organism S. pyogenes (MIC $0.5 \mathrm{mg} / \mathrm{L}$ ) - whereas $34.5 \%$ achieved the target against $H$. influenzae (MIC $2 \mathrm{mg} / \mathrm{L}$ ). With the dosing interval extended to 6 hours, the pharmacodynamic target ( $70 \%$ fT $>$ MIC) could be achieved in $86.6 \%$ of patients against $S$. pyogenes (MIC $0.5 \mathrm{mg} / \mathrm{L}$ ) and $59.3 \%$ against 
Table 2 Population pharmacokinetic parameters of cefathiamidine and bootstrap results

\begin{tabular}{|c|c|c|c|c|}
\hline \multirow[t]{2}{*}{ Parameters } & \multicolumn{2}{|l|}{ Full data set } & \multicolumn{2}{|c|}{ Bootstrap } \\
\hline & Final estimate & RSE (\%) & Median & 5th-95th \\
\hline \multicolumn{5}{|l|}{$C L(L / h)$} \\
\hline \multicolumn{5}{|c|}{$C L=\theta \mid \times(C W / 17.75)^{0.75} \times \operatorname{EXP}\left(\eta_{1}\right)$} \\
\hline$\Theta \mathrm{I}$ & 3.93 & 6.90 & 3.93 & $3.47-4.42$ \\
\hline \multicolumn{5}{|l|}{ VI (L) } \\
\hline \multicolumn{5}{|c|}{$\mathrm{V}=\theta 2 \times(\mathrm{CW} / \mathrm{I7.75})$} \\
\hline$\Theta 2$ & 4.18 & 7.80 & 4.11 & $3.11-4.78$ \\
\hline \multicolumn{5}{|l|}{$\mathrm{Q}(\mathrm{L} / \mathrm{h})$} \\
\hline \multicolumn{5}{|c|}{$\mathrm{Q}=\theta 3 \times(\mathrm{CW} / / \mathrm{I.75})^{0.75}$} \\
\hline$\Theta 3$ & 0.50 & 30.90 & 0.51 & $0.25-1.75$ \\
\hline \multicolumn{5}{|c|}{ V2 (L) } \\
\hline \multicolumn{5}{|c|}{$\mathrm{V}=\theta 4 \times(\mathrm{CW} / 17.75) \times \mathrm{EXP}\left(\eta^{2}\right)$} \\
\hline$\Theta 4$ & 1.30 & 33.50 & 1.45 & $0.77-2.68$ \\
\hline \multicolumn{5}{|c|}{ Interindividual variability (\%) } \\
\hline $\mathrm{CL}$ & 41.83 & 30.0 & 39.62 & $29.14-51.38$ \\
\hline $\mathrm{V} 2$ & 74.23 & 65.2 & 74.70 & $40.82-123.69$ \\
\hline \multicolumn{5}{|c|}{ Residual variability (\%) } \\
\hline ERR (I) & 35.78 & 16.60 & 35.07 & $28.87-40.34$ \\
\hline
\end{tabular}

Note: In our population, $17.8 \mathrm{~kg}, 4.9$ years, and $193 \mathrm{~mL} / \mathrm{min}$ are the median current weight (day of the study), age, and creatinine clearance, respectively.

Abbreviations: VI, central volume of distribution; V2, peripheral volume of distribution; $\mathrm{Q}$, intercompartmental clearance; $\mathrm{CL}$, clearance.

H. influenzae (MIC $2 \mathrm{mg} / \mathrm{L})$. However, the extended dosing regimen $(100 \mathrm{mg} / \mathrm{kg} /$ day four times daily) results in only $25.0 \%$ of children achieving the pharmacodynamic target against MSSA (MIC $8 \mathrm{mg} / \mathrm{L})$ and indicates that a higher dosing regimen is required (Figure 5).

\section{Discussion}

This is the first model developed to describe the pharmacokinetics of cefathiamidine in children. Our results show that a two-compartment model with first-order elimination was optimal for pharmacokinetic data modeling.

As cefathiamidine is almost exclusively eliminated by the renal route, both renal function and size should have

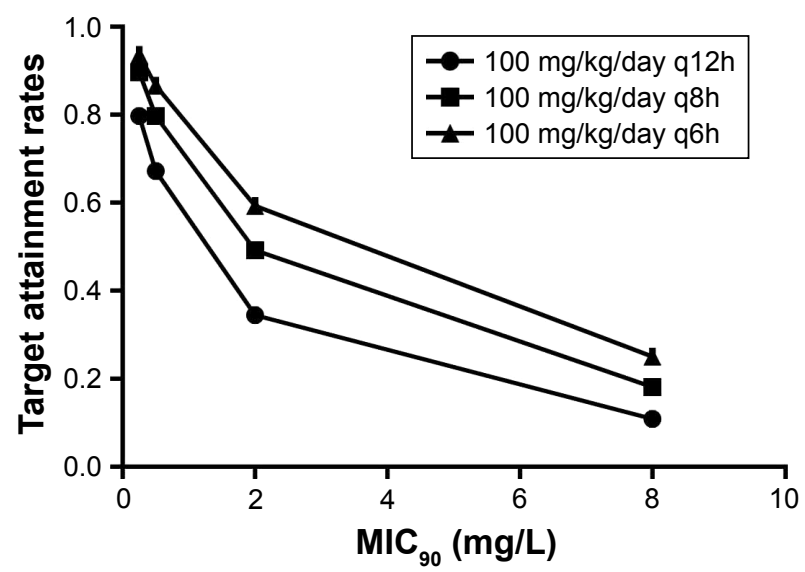

Figure 4 Probability of target $(70 \% \mathrm{fT}>\mathrm{MIC})$ attainment for cefathiamidine $100 \mathrm{mg} / \mathrm{kg} /$ day q $\mathrm{2h}$, q8h, and q6h against target organisms in children $(2 \leq$ age $<12)$. important influences on the dosing regimen in children. ${ }^{18}$ Covariate analysis identified that body weight significantly influenced cefathiamidine clearance, whereas creatinine clearance did not. This can be explained by the limited range of creatinine clearance $(130-462 \mathrm{~mL} / \mathrm{min})$. None of the included patients had insufficient renal function. The plot of cefathiamidine versus creatinine clearance did not show any trend. It should be noted that most of the children included in this study had malignant hematologic disease and represent a critical population in whom disease may also impact pharmacokinetics due to hyperfiltration, along with the maturation. ${ }^{19}$ The estimated vancomycin ${ }^{20,21}$ and teicoplanin ${ }^{22} \mathrm{CLs}$ in children with malignant hematologic

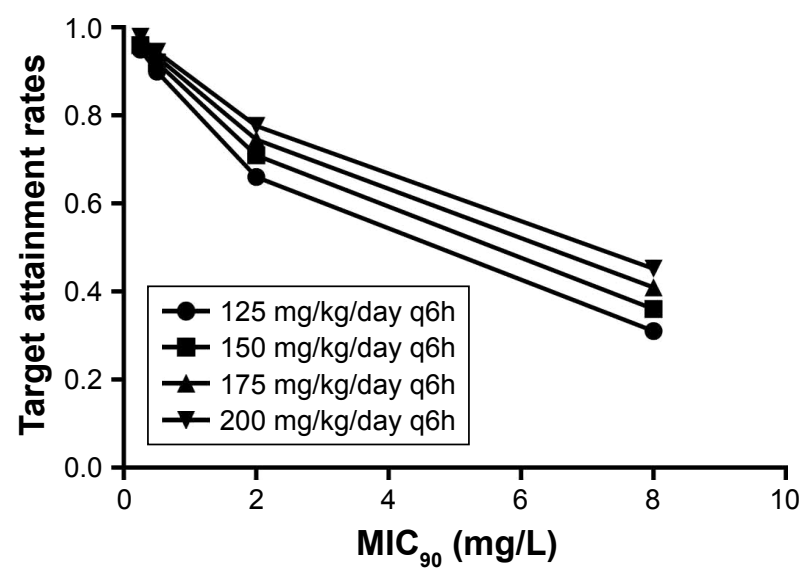

Figure 5 Probability of target $(70 \% \mathrm{fT}>\mathrm{MIC})$ attainment for cefathiamidine $125, \mathrm{I}$, 175 , and $200 \mathrm{mg} / \mathrm{kg} /$ day $q 6 \mathrm{~h}$ against target organisms in children $(2 \leq$ age $<12)$. 
Table 3 Pharmacokinetics of cefathiamidine obtained from different studies

\begin{tabular}{|c|c|c|c|}
\hline \multirow[t]{2}{*}{ Parameter } & \multicolumn{3}{|c|}{ Value for the group (reference or source) } \\
\hline & $\begin{array}{l}\text { Adults } \\
\text { reference } \\
5 \text { mean }\end{array}$ & $\begin{array}{l}\text { Adults } \\
\text { reference } \\
6 \text { mean }\end{array}$ & $\begin{array}{l}\text { Children with malignant } \\
\text { hematologic disease (this } \\
\text { study), median (range) }\end{array}$ \\
\hline No of patients & 9 & 7 & 54 \\
\hline Age (year) & $22.5 \pm 1.1$ & Adults & $4.9(2.0-11.8)$ \\
\hline Weight (kg) & $61.7 \pm 6.2$ & $47-68$ & $17.8(8.0-36.0)$ \\
\hline CL (liters/h/kg) & $0.17 \pm 0.02$ & $0.15 \pm 0.035$ & $0.25(0.05-0.43)$ \\
\hline V (liters/kg) & $0.11 \pm 0.02^{\mathrm{a}}$ & $0.59 \pm 0.40$ & $0.31(0.26-0.38)$ \\
\hline
\end{tabular}

Note: ${ }^{a}$ Volume of the central compartment.

disease were higher than in the general nonselected pediatric populations. The estimated cefathiamidine CL and V in this study and the reported values in general adult patients are summarized in Table 3.5,6

The time of free antimicrobial drug concentration above the minimum inhibitory concentration was regarded as a surrogate marker of therapeutic efficacy for cephalosporin. A target of $40 \%-50 \% \mathrm{fT}>\mathrm{MIC}$ is generally accepted for cephalosporin in adult patients. ${ }^{23,24}$ However, a higher fT $>$ MIC target of $70 \%-80 \%$ is recommended to ensure efficacy and avoid the induction of antibiotic resistance for the critically ill and with the immunocompromised status of pediatric population. ${ }^{25-28}$ As shown in the simulation results, the currently used dosing regimen $(100 \mathrm{mg} / \mathrm{kg} /$ day q12h) could have approximately $34.5 \%$ children achieving the pharmacodynamic target against $H$. influenzae (MIC $2 \mathrm{mg} / \mathrm{L}$ ). To achieve maximal bactericidal activity, an increased dose and/or dosing frequency were necessary. As the safety of a higher dose has not been tested, the option of an increased frequency was considered. An optimal dosing regimen of $100 \mathrm{mg} / \mathrm{kg} /$ day q6h was required against H. influenza (MIC $2 \mathrm{mg} / \mathrm{L}$ ). For more resistant bacterial strains (eg, MSSA, MIC $8 \mathrm{mg} / \mathrm{L}$ ), a different antimicrobial drug should be considered in clinical practice.

Our study had some limitations. Most of the children included in this study had malignant hematologic disease. The clearance of cefathiamidine might be augmented due to hyperfiltration. The pharmacokinetics of cefathiamidine in the "general" pediatric population still needs to be evaluated. In addition, the pharmacokinetic model of cefathiamidine was only internally validated. External validation was not undertaken because of the limited number of patients. Ultimately, an optimal dose regimen based on modeling and simulation should be evaluated in clinical practice to confirm its clinical benefits.

\section{Conclusion}

A population pharmacokinetic model of cefathiamidine was developed in children. Body weight had a significant impact on cefathiamidine clearance. The currently used dose regimen of $100 \mathrm{mg} / \mathrm{kg} /$ day q $12 \mathrm{~h}$ is associated with a high risk of underdosing in pediatric patients. To reach the target $70 \%$ $\mathrm{fT}>\mathrm{MIC}$, a dosage of $100 \mathrm{mg} / \mathrm{kg} /$ day q6h is required against H. influenzae (MIC $2 \mathrm{mg} / \mathrm{L})$. Until the safety of a higher dose of cefathiamidine is tested in studies, other antibiotics should be considered for more resistant bacterial strains (eg, MSSA). The model-based dosing regimen of cefathiamidine in children was established on the basis of population pharmacokinetic-pharmacodynamic analysis.

\section{Acknowledgments}

This study was supported by the Science and Technology Planning Project of Hebei Province (grant no 15277705D); Health and Family Planning Commission of Hebei Province (grant no 20150100); Scientific Research Foundation for the High-Level Returned Overseas Chinese Scholars (grant no CG2016030001; Ministry of Human Resources and Social Security); and the Hundred-Talent Program (grant no E2015100010; The People's Government of Hebei Province).

Preliminary results of this study were presented at the 16th meeting of the European Society for Developmental Perinatal and Paediatric Pharmacology Congress, 2017, Leuven, Belgium. Li-Juan Zhi and Li Wang are co-first authors.

\section{Disclosure}

All authors have completed the Unified Competing Interest form (available on request from the corresponding author). None of the authors have any other relationships or activities that could appear to have influenced the submitted work.

\section{References}

1. National pediatric multi-center cooperative group of cefathiamidine observation. Effects of cefathiamidine on the treatment of children with acute bacterial infectious disease. Zhonghua Er Ke Za Zhi. 2003;41(1):54-55.

2. Zhang W, Shen X, Bergman U, et al. Drug utilisation 90\% (DU90\%) profiles of antibiotics in five Chinese children's hospitals (2002-2006). Int $J$ Antimicrob Agents. 2008;32(3):250-255.

3. Zhang W, Shen X, Wang Y, et al. Antibiotic use in five children's hospitals during 2002-2006: the impact of antibiotic guidelines issued by the Chinese Ministry of Healthy. Pharmacoepidemiol Drug Saf. 2008;17(3):306-311.

4. Chen HY, Williams JD. The killing effects of cefathiamidine or ampicillin alone and in combination with gentamicin against enterococci. J Antimicrob Chemother. 1983;12(1):19-26.

5. Tze-ying T, Fu W, Chih-lin C, et al. Clinical and laboratory studies of a new cephalosporin derivative - cefathiamidine. Chin Med J (Engl). 1979; 92(1):26-36. 
6. Hu M, Hu CQ. Identification of the degradation compounds of cefathiamidine by liquid chromatography-tandam mass spectrometry. Yao Xue Хие Bao. 2006;41(10):1015-1019.

7. Hooker AC, Staatz CE, Karlsson MO. Conditional weighted residuals (CWRES): a model diagnostic for the FOCE method. Pharm Res. 2007;24(12):2187-2197.

8. Brendel K, Comets E, Laffont C, Laveille C, Mentré F. Metrics for external model evaluation with an application to the population pharmacokinetics of gliclazide. Pharm Res. 2006;23(9):2036-2049.

9. Bergstrand M, Hooker AC, Wallin JE, Karlsson MO. Predictioncorrected visual predictive checks for diagnosing nonlinear mixedeffects modes. AAPS J. 2011;13(2):143-151.

10. Comets E, Brendel K, Mentré F. Computing normalised prediction distribution errors to evaluate nonlinear mixed-effect models: the npde add-on package for R. Comput Methods Programs Biomed. 2008; 90(2):154-166.

11. Craig WA. Pharmacokinetic/pharmacodynamic parameters: rationale for antibacterial dosing of mice and men. Clin Infect Dis. 1998;26(1): 1-10; quiz 11-12.

12. Dudley MN, Ambrose PG. Pharmacodynamics in the study of drug resistance and establishing in vitro susceptibility breakpoints: ready for prime time. Curr Opin Microbiol. 2000;3(5):515-521.

13. Bradley JS, Sauberan JB, Ambrose PG, Bhavnani SM, Rasmussen MR, Capparelli EV. Meropenem pharmacokinetics, pharmacodynamics, and Monte Carlo simulation in the neonate. Pediatr Infect Dis J. 2008; 27(9):794-799.

14. Guangzhou Baiyunshan Pharmaceuticals. October 2010. Xianlisu (cefathiamidine for injection) prescribing information. Guangzhou Baiyunshan Pharmaceuticals, Guangzhou, People's Republic of China.

15. Drusano GL. Prevention of resistance: a goal for dose selection for antimicrobial agents. Clin Infect Dis. 2003;36(Suppl 1):S42-S50.

16. Visser LG, Arnouts P, van Furth R, Mattie H, van den Broek PJ. Clinical pharmacokinetics of continuous intravenous administration of penicillins. Clin Infect Dis. 1993;17(3):491-495

17. Lan AJ, Colford JM, Colford JM Jr. The impact of dosing frequency on the efficacy of 10-day penicillin or amoxicillin therapy for streptococcal tonsillopharyngitis: a meta-analysis. Pediatrics. 2000; 105(2):E19.
18. Rodieux F, Wilbaux M, van den Anker JN, Pfister M. Effect of kidney function on drug kinetics and dosing in neonates, infants, and children. Clin Pharmacokinet. 2015;54(12):1183-1204.

19. Zhao W, Fakhoury M, Fila M, Baudouin V, Deschênes G, JacqzAigrain E. Individualization of valganciclovir prophylaxis for cytomegalovirus infection in pediatric kidney transplant patients. Ther Drug Monit. 2012;34(3):326-330.

20. Zhao W, Zhang D, Fakhoury M, et al. Population pharmacokinetics and dosing optimization of vancomycin in children with malignant hematological disease. Antimicrob Agents Chemother. 2014; 58(6):3191-3199.

21. Chang D. Influence of malignancy on the pharmacokinetics of vancomycin in infants and children. Pediatr Infect Dis J. 1995;14(8):667-673.

22. Zhao W, Zhang D, Storme T, Baruchel A, Declèves X, Jacqz-Aigrain E. Population pharmacokinetics and dosing optimization of teicoplanin in children with malignant haematological disease. Br J Clin Pharmacol. 2015;80(5):1197-1207.

23. Craig WA. Interrelationship between pharmacokinetics and pharmacodynamics in determining dosage regimens for broad-spectrum cephalosporins. Diagn Microbiol Infect Dis. 1995;22(1-2):89-96.

24. Craig WA. Antimicrobial resistance issues of the future. Diagn Microbiol Infect Dis. 1996;25(4):213-217.

25. Craig WA. Basic pharmacodynamics of antibacterials with clinical applications to the use of beta-lactams, glycopeptides, and linezolid. Infect Dis Clin North Am. 2003;17(3):479-501.

26. Lutsar I, Metsvaht T. Understanding pharmacokinetics/pharmacodynamics in managing neonatal sepsis. Curr Opin Infect Dis. 2010; 23(3):201-207

27. van den Anker JN. Population pharmacokinetics and dosing of amoxicillin in (pre)term neonates. Ther Drug Monit. 2006;28(6):816; author reply $816-817$.

28. Leroux S, Roué JM, Gouyon JB, et al. A population and developmental pharmacokinetic analysis to evaluate and optimize cefotaxime dosing regimen in neonates and young infants. Antimicrob Agents Chemother. 2016;60(11):6626-6634.
Drug Design, Development and Therapy

\section{Publish your work in this journal}

Drug Design, Development and Therapy is an international, peerreviewed open-access journal that spans the spectrum of drug design and development through to clinical applications. Clinical outcomes, patient safety, and programs for the development and effective, safe, and sustained use of medicines are the features of the journal, which

\section{Dovepress}

has also been accepted for indexing on PubMed Central. The manuscript management system is completely online and includes a very quick and fair peer-review system, which is all easy to use. Visit http://www.dovepress.com/testimonials.php to read real quotes from published authors. 